\title{
Stromal Signatures of Breast Carcinogenesis in Fibroadenoma-A Pilot Study
}

Rani Kanthan ${ }^{1 *}$, Jenna-Lynn Senger ${ }^{1}$ and Kanthan SC ${ }^{2}$

${ }^{1}$ Department of Pathology and Laboratory Medicine, Canada

${ }^{2}$ Department of Surgery, University of Saskatchewan, Canada

\begin{abstract}
Background: Fibroadenoma ( $F A)$ is a benign breast neoplasm associated with an increased risk of subsequent carcinogenesis; yet, the relationship between these two events remains undetermined. Traditional breast paradigms that focus on the epithelial cell as the primary origin of cancer need to be revisited. Our working hypothesis is that, stromal dysfunction through epithelial-stromal 'cross-talk' in the host microenvironment is perhaps the earliest initiating event in breast carcinogenesis.
\end{abstract}

Methods: 60 cases of randomly selected FA were analyzed in this study. Paraffin embedded tissue samples were analyzed using the antibodies TAG72, ErbB2, p53, CD10, Ki67, Bcl2, CD31, Nestin, and Laminin. Stromal and epithelial components were scored and compared on a semi-quantitative scale.

Results: Six of the 60 cases subsequently developed breast cancer between 1-12 years after the diagnosis of FA. Comparison of the stromal:epithelial (S:E) ratio of immunohistochemical scores yielded four significant trends suggestive of stromal dysfunction. In pre-cancerous lesions, $\mathrm{S}$ : $\mathrm{E}$ ratios of non-cancerous vs. pre-cancerous FA were lower for a) CD10 at 40:100 vs. $15: 95(p=0.045), b) B c 12$ at 40:85 vs. 20:80 ( $p=0.048), c)$ CD31 at 30:0 vs. 9:0 $(p=0.0156)$ and $d)$ Nestin at $40: 80$ vs. $15: 80(p=0.013)$.

Conclusion: This preliminary study suggests early alterations in the stroma precede development of the epithelial phenotype of breast cancer. Improved understanding of host stromal microenvironmental changes can lead to the recognition of 'stromal signatures' as risk assessment markers in FA for future breast carcinogenesis. In the future, exploration in both benign and malignant proliferations of breast lesions should be undertaken to further elucidate these 'stromal signatures' in breast cancer for men and women.

Keywords: Fibroadenoma; Stroma; Stromal-Epithelial Crosstalk; CD10; CD31; Bcl-2; Nestin

\section{Introduction}

Despite recent research linking the microenvironment to the development of breast malignancies, our understanding of the genes, proteins, and pathways regulating the stromal-epithelial interactions in normal and neoplastic breast tissue remains scarce. The traditional hypothesis of altercation in epithelial cells resulting in their uncontrolled growth is insufficient in fully explaining the carcinogenesis and the complexity of tumor progression [1]. Instead, emerging studies suggests that changes in the interactions between the mammary stroma and epithelium may be an initiator of tumorigenesis. As such, genetic and epigenetic changes that result in carcinogenesis/ tumorigenesis may occur not only in the epithelial component, but also in the stroma. This model of tumor development though explored theoretically and in limited animal studies, there remains a paucity of studies on human tissues to support this hypothesis.

Fibroadenoma (FA) is a benign fibroepithelial lesion that arises most commonly in young women as a solitary mobile, painless lump. Carcinomatous lesions have been shown to arise within an existing FA or delayed at more than twenty years after the initial diagnosis in 0.12 $0.3 \%$ of FAs [2]. Fibroadenoma was chosen as an initial tumour for this pilot study as it is basically a stromal neoplasm with a benign clinical behaviour. Atypical tumour-stromal fibroblasts have been increasingly recognized as important histological outcome predictors for patients with invasive ductal carcinoma of the breast. The histological features of such atypical tumour-stromal fibroblasts include cells with a) increased number of nuclei, b) increased nuclear size, c) irregular nuclear shape, d) the presence of nucleoli and e) multiple nuclei resulting possibly from fusion of cells [3,4]. Additionally, atypical features of tumour cells and stromal cells have also been described in vessels and lymph nodes as important prognostic parameters in non-breast cancers such as extrahepatic bile duct carcinoma [5]. Our hypothesis is that such histologic phenotypical changes is preceded by alterations at a molecular/genetic/epigenetic level similar to the accepted multifactorial pathway of carcinogenesis in colorectal carcinoma. In this retrospective study, therefore, we analyzed tissue samples obtained from sixty patients diagnosed with fibroadenoma in terms of its expression of ten specifically-chosen antibodies that we believe may be dominant participants in the stromal-epithelial crosstalk.

\section{Materials and Methods}

\section{Patients and tissue specimens}

Sixty cases of fibroadenoma were identified using the computerbased Laboratory Information System (LIS) of the Saskatoon Health Region between the years 1997-1999. This timeframe was chosen

*Corresponding author: Dr. Rani Kanthan, Royal University Hospital, Room 2868 G-Wing, 103 Hospital Drive, Saskatoon, SK Canada, S7N 0W8, Tel: 306-6552158; Fax: 306-655-2223; E-mail: rani.kanthan@saskatoonhealthregion.ca

Received August 24, 2011; Accepted November 19, 2011; Published November 22, 2011

Citation: Kanthan R, Senger JL, Kanthan SC (2011) Stromal Signatures of Breast Carcinogenesis in Fibroadenoma-A Pilot Study. J Carcinogene Mutagene 2:123. doi:10.4172/2157-2518.1000123

Copyright: (c) 2011 Kanthan R, et al. This is an open-access article distributed under the terms of the Creative Commons Attribution License, which permits unrestricted use, distribution, and reproduction in any medium, provided the original author and source are credited. 
because the LIS database begins in January 1997, and we wished to optimize the length of the follow-up period (1998-2010, 12 years). Patients ranged in age from 20-78 years, with a mean of 40.5 years (median 41 years). 33 fibroadenomas were in the left breast, and 27 were in the right. Patients' medical records were examined to complete a 12-year follow-up, and any breast pathologies that had developed since the diagnosis of FA were retrieved for analysis and review. The patients that later developed breast cancer was identified into a separate 'test group', and those that did not develop cancer within this time acted as a control group.

All specimens collected, as per laboratory policy, had been fixed with $4 \%$ formalin for periods no longer than 24 hours and embedded in paraffin. The tissue blocks had been sectioned and stained with hematoxylin and eosin. All histopathological material in the identified groups was reviewed to confirm the diagnosis of fibroadenoma and breast cancer respectively.

\section{Immunohistochemistry}

From the available material, a representative tissue block was chosen, and sections of $3 / 4 \mu \mathrm{m}$ thickness were cut and placed on glass slides coated with 4-aminopropyl triethoxysilane for immunohistochemistry studies. Immunohistochemical staining was performed using an autoimmunostainer with autoclave antigenretrieval. Each specimen was immersed in citrate buffer and incubated at $121^{\circ} \mathrm{C}$ for 10 minutes. Immunoperoxidase staining was performed using a labelled streptavidin-biotin technique for each of the markers. The primary antibodies analyzed in this study including the company, clone and dilutions used are summarized in Table 1. The entire tumour represented in the section was examined and assessed. In each case, the stroma of the fibroadenoma and the adjacent non-fibroadenomatous stroma were analyzed. As negative controls, the primary antibody was replaced with normal mouse immunoglobulin and the primary antibody solution was substituted with a phosphate-buffered saline (PBC) as per laboratory protocol. Immunostained slides were graded on a $0-100 \%$ scale based on the number and intensity of the positive cells stained. Slides were scored at medium-high power with an average percentage being taken after counting representative cells over 3-4 regions.

\section{Statistical analysis}

Results were grouped based on if the patient later developed cancer or not with the non-cancerous FAs as the 'control' group and the precancerous FAs as the 'test' group. Within each group, and for each antibody an S:E ratio (\% stromal staining: \% epithelial staining) was found. Using the statistical software SPSS for Windows, version 17.0 the two groups were compared using a paired $t$-test. A p-value of $<0.05$ was considered statistically significant.

\section{Results}

Sixty cases of fibroadenoma were identified between 1997 and 1999. Of these, six (10\%) subsequently developed breast cancer within the twelve-year period (1998-2010). A summary of the demographics of patients that subsequently developed cancer is outlined in Table 2. Patients' ages ranged from 33-52, with a median of 42.5 years. Four (67\%) of the patients developed cancer in the same breast as the fibroadenoma, and $2(33 \%)$ were in the opposite breast. The time interval between the diagnosis of fibroadenoma and cancer was most

\begin{tabular}{|l|l|l|}
\hline Antigen & Dilution & Company \& Clone \\
\hline Cerb2 & $1 / 1600$ & Microwave AR in 1mM EDTA pH9.0 \\
\hline Cerb2 SP3 & $1 / 200$ & Microwave AR in 1mM EDTA pH9.0 \\
\hline P53 & $1 / 100$ & Microwave AR in 1mM EDTA pH9.0 \\
\hline TAG72 & $1 / 200$ & Microwave AR in 1mM EDTA pH9.0 \\
\hline CD10 & $1 / 20$ & Microwave AR in 1mM EDTA pH9.0 \\
\hline Ki67 & $1 / 50$ & Microwave AR in 1mM EDTA pH9.0 \\
\hline Bcl-2 & $1 / 20$ & Microwave AR in 1mM EDTA pH9.0 \\
\hline CD31 & $1 / 10$ & Microwave AR in 1mM EDTA pH9.0 \\
\hline Laminin & $1 / 100$ & Pepsin AR, Sigma clone LAM-89 \\
\hline Nestin & $1 / 50$ & Microwave AR in 1 mM EDTA pH9.0, Abcam polyclonal \\
\hline
\end{tabular}

Table 1: Antibodies used in immunohistochemical staining.

\begin{tabular}{|l|l|l|l|l|l|l|}
\hline \multirow{2}{*}{ Case \# } & \multirow{2}{*}{ Age } & \multicolumn{2}{|c|}{ Fibroadenoma } & \multicolumn{3}{c|}{ Cancer } \\
\cline { 3 - 8 } & & Year & Laterality & Year & Laterality & \multicolumn{1}{c|}{ Diagnosis } \\
\hline 1 & 52 & 1997 & Right & 2004 & Left & Ductal carcinoma \\
\hline 2 & 50 & 1997 & Right & 1997 & Right & Lobular carcinoma \\
\hline 3 & 45 & 1997 & Right & 1998 & Right & Ductal carcinoma \\
\hline 4 & 40 & 1997 & Right & 2009 & Left & Ductal carcinoma \\
\hline 5 & 33 & 1997 & Right & 1997 & Right & Ductal carcinoma \\
\hline 6 & 33 & 1997 & Left & 1997 & Left & Lobular carcinoma \\
\hline
\end{tabular}

Table 2: Demographics of patients that developed breast cancer.

commonly within a year ( $50 \%$ cases), though diagnosis as late as twelve years was found. The most common histological phenotype of the breast cancer was ductal carcinoma in two-thirds (67\%) with the remaining third being lobular carcinoma.

Comparison of the stromal: epithelial (S:E) ratio of immunohistochemical scores yielded four significant trends.

CD10: The extent of staining in the non-cancerous epithelial component ranged from $60-100 \%$ and in the stromal component from $10-80 \%$. In the precancerous lesions, epithelial staining ranged $90-$ $100 \%$ and stromal $10-20 \%$. The median S:E for the non-cancerous FA was 40:100 versus the cancerous at 15:95 ( $\mathrm{p}=0.042$ ) (Figure 2).

Bcl2: The extent of staining in the non-cancerous epithelial component ranged from $10-100 \%$ and in the stromal component from $5-80 \%$. In the precancerous lesions, epithelial staining ranged $80-90 \%$ and stromal 20-30\%. The median S:E for the non-cancerous FA was at 40:85 versus the cancerous at 20:80 ( $\mathrm{p}=0.047)$ (Figure 3$)$.

CD31: There was consistently no staining in the non-cancerous epithelial tissue, and the extent of stromal staining ranged from $10-40 \%$. In the precancerous lesions, there was no epithelial CD31 staining, and stromal staining was identified in 5-10\% of cells. The median S:E for the non-cancerous FA was at 30:0 versus the cancerous at 10:0 ( $\mathrm{p}=0.015)$ (Figure 4).

Nestin: The extent of staining in the non-cancerous epithelial component ranged from $40-100 \%$ and in the stromal component from $10-80 \%$. In the precancerous lesions, epithelial staining ranged 40 $100 \%$ and stromal $0-30 \%$. The median S:E for the non-cancerous FA was at $40: 80$ vs. $15: 80$ in the pre-cancerous ( $\mathrm{p}=0.013$ ) (Figure 5).

There was no significant change in the epithelial or stromal expression of TAG72, ErbB2, p53, Ki67 or laminin when comparing the fibroadenomas that subsequently developed cancer with those that did not over a 12-year follow-up period. The stromal expression of all the proteins described above was similar in both the fibroadenomatous 


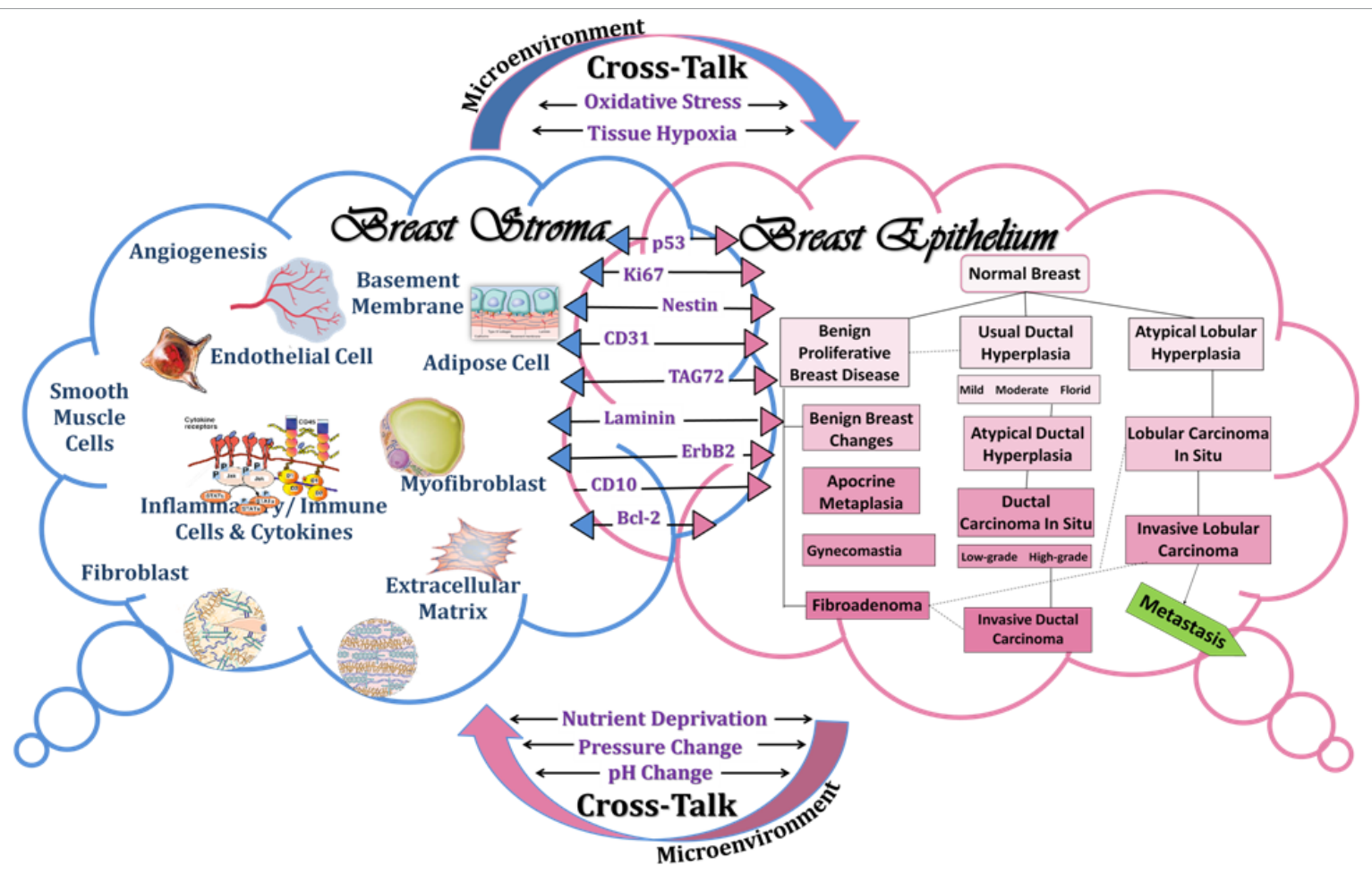

Figure 1: Schematic outline of the stromal-epithelial cross-talk in the breast microenvironment. On the left-hand side of the diagram within the blue bubble is depicted the normal constituents of the stroma that includes blood vessels, basement membrane, myofibroblasts, extracellular matrix, fibroblasts, inflammatory/immune cells, smooth muscle cells and adipose cells. On the right-side within the pink bubble is the normal breast epithelial cell illustrating the traditionally-held stepwise linear progression to breast cancer. The central 'ven' of these bubbles is the pictorial representation of the stromal-epithelial cross-talk between the nine proteins ( 553 , Ki67, nestin, CD31, TAG72, laminin,ErbB2, CD10, Bcl-2) that were studied. The incipient factors that modulate the microenvironment such as oxidative stress, tissue hypoxia, nutrient deprivation, and pressure/pH changes are figuratively represented at the outskirts of the 'ven'.

and non-fibroadenomatous breast tissues in each case. Figure 6 is a composite photomicrograph that demonstrates expression of CD10, Bcl-2, CD31 and Nestin in the stromal and epithelial cells of the breast microenvironment.

\section{Discussion}

Historically, breast cancer research on carcinogenesis has focused on the epithelial changes that precede tumour development in an effort to explain the pathogenesis of this disease. Recent studies suggest

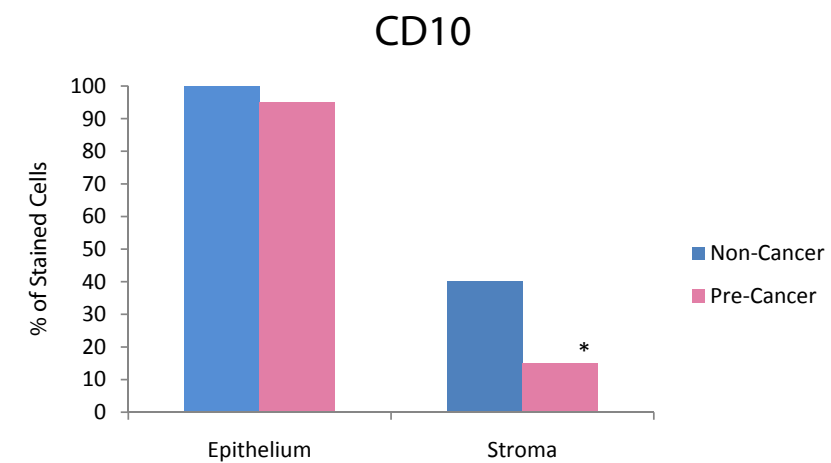

Figure 2: This bar graph shows CD10 protein expression in the epithelium and stroma between the pre-cancerous(pink) and non-cancerous(blue) fibroadenomas. The $y$-axis is the percentage of positively stained cells in multiples of 10 from $0-100 \%$. The * indicates statistical significance of a p-value $<0.05$.

\section{$\mathrm{BCl}-2$}

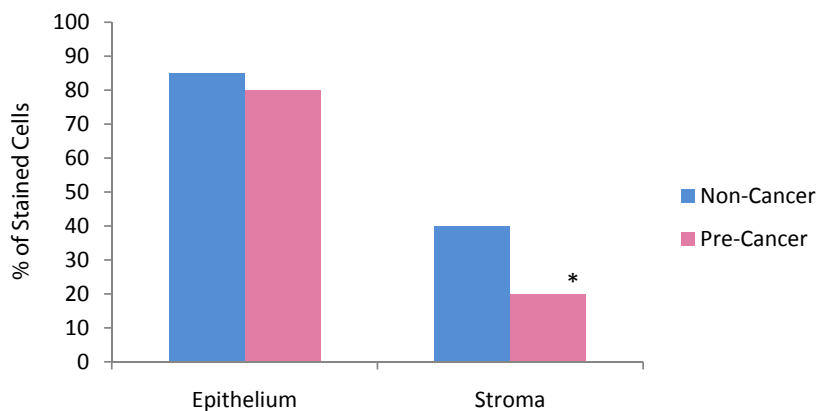

Figure 3: This bar graph shows $\mathrm{Bcl}-2$ protein expression in the epithelium and stroma between the pre-cancerous(pink) and non-cancerous(blue) fibroadenomas. The $y$-axis is the percentage of positively stained cells in multiples of 10 from $0-100 \%$. The * indicates statistical significance of a p-value $<0.05$.

'thinking outside of the cell', as an emerging concept with the stroma being partially responsible for breast carcinogenesis. The stroma occupies over $80 \%$ of the volume of the resting breast and comprises the area around the epithelial cells. It consists of fibroblasts, myofibroblasts, glial, fat, immune, vascular, lymphovascular, endothelial, and smooth muscle cells as well as the extracellular matrix, the basement membrane and soluble proteins such as growth factors, cytokines, and hormones [1,6-8]. Changes to this microenvironment, such as oxidative stress, 
tissue hypoxia, nutrient deprivation, and changes in pressure or $\mathrm{pH}$ have been shown to increase the rate of DNA mutations, thus causing genetic instability in these stromal cells [7]. Multiple genetic events causing oncogene-activation and disruption of the tumour suppressor genes have been identified in breast cancer progression. Commonly identified genetic changes include gain-of-function mutations in proto-oncogenes, loss-of-function mutations in tumour suppressor genes, and epigenetic deregulation [9]. Such alterations may occur not only in the epithelial cells, but also in components of the stroma, creating an environment conducive to cancer growth. The tumour microenvironment, composed of cancer cells, stressed normal cells, stromal tissue, and extracellular matrix, has been implicated in the progression, invasion, and spread of cancer. Carcinogenetic characteristics include sustaining proliferative signals, evading growth suppressors, resisting cell death, enabling replicative immortality, inducing angiogenesis, activating invasion/metastasis, reprogramming energy metabolism, and evading immune destruction. We believe alterations in this host microenvironment modify its function and phenotype with disruption of the epithelial-stromal cross-talk resulting in neoplastic initiation. A schematic representation of the stromalepithelial crosstalk in breast microenvironment is illustrated in Figure 1.

\section{CD31}

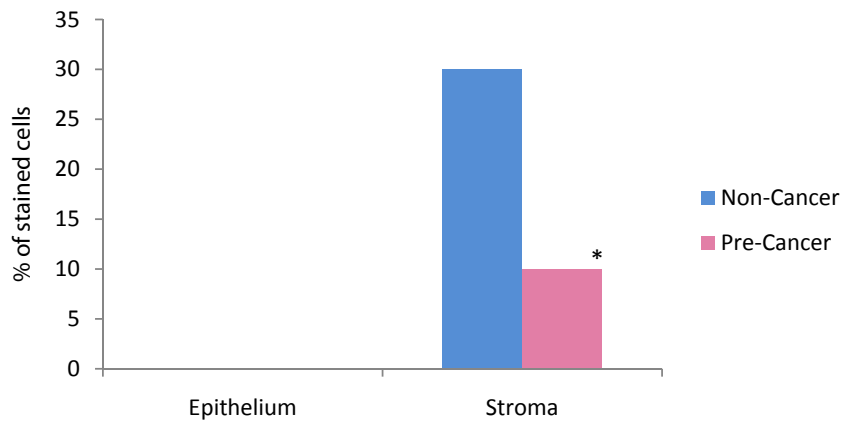

Figure 4: This bar graph shows CD31 protein expression in the epithelium and stroma between the pre-cancerous(pink) and non-cancerous(blue) fibroadenomas. The $y$-axis is the percentage of positively stained cells in multiples of 10 from $0-100 \%$. The * indicates statistical significance of a p-value $<0.05$.

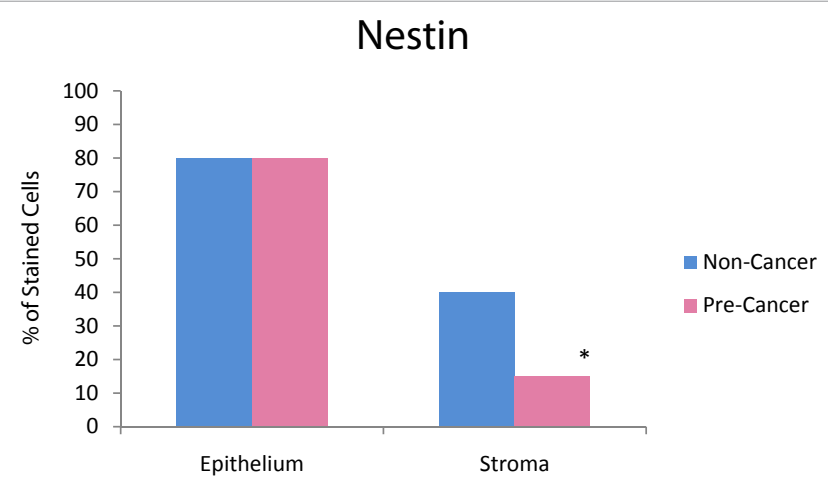

Figure 5: This bar graph shows Nestin protein expression in the epithelium and stroma between the pre-cancerous(pink) and non-cancerous(blue) fibroadenomas. The $y$-axis is the percentage of positively stained cells in multiples of 10 from $0-100 \%$. The * indicates statistical significance of a p-value $<0.05$.

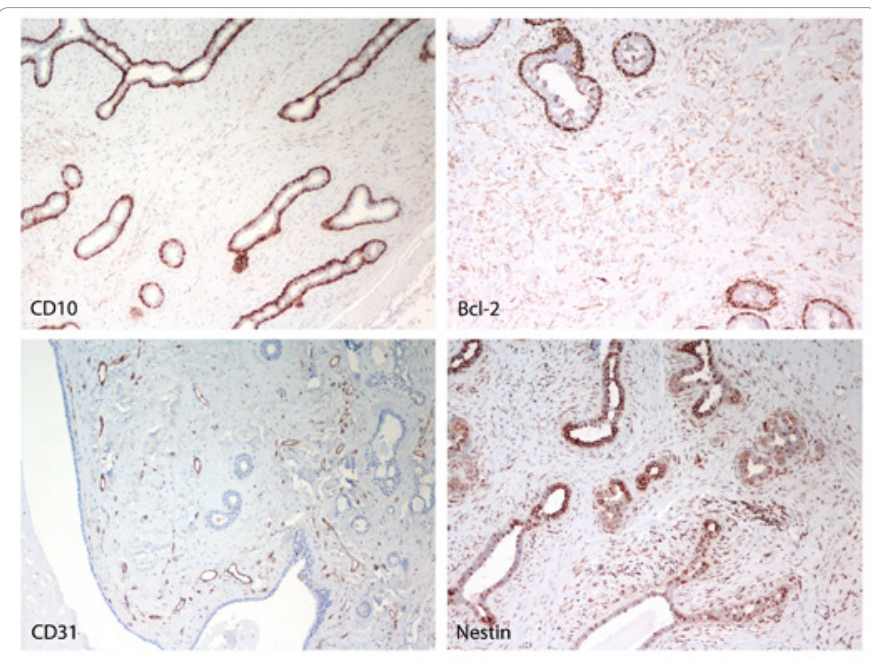

Figure 6: Composite of photomicrographs at medium magnification demonstrating expression of CD10, Bcl-2, CD31, and nestin in the stromal and epithelial components of the breast microenvironment.

To evaluate stromal dysregulation in patients with fibroadenoma, we examined the expression of nestin, laminin, CD10, Bcl-2, CD31, ErbB2, p53, TAG-72, and Ki67 in both the epithelial and stromal components of fibroadenomas in the identified pre-cancerous test group and in the non-cancerous control group. We found statistically significant changes in the expression of CD10, Bcl-2, CD31, and nestin between patients who later developed a breast malignancy compared to those that did not. These four antibodies are herein discussed.

CD10 is a cell surface zinc-dependent metalloprotease reported to be expressed in the stroma of prostate, breast, colorectal and lung carcinomas [10]. This protein functions as a peptidase that cleaves proteins from the ECM, resulting in tissue remodeling. CD10 is expressed by the breast's myoepithelial cells during development and after maturation; therefore, this protease is considered a specific myoepithelial cell marker. The stroma of the developing breast is not positive for CD10. Stromal cells in fibroadenomas have demonstrated CD10 positivity [11], and expression has been observed in the stroma and ECM of breast cancers [10,11]. It is hypothesized CD10 may craft a specialized microenvironment to facilitate cancer cell invasion, and/ or may cross-talk with cancerous cells to speed up the cell cycle and promote cell mobility.

CD10 is believed to represent a stronger predictive factor for both tumour recurrence and death than node status, tumour size, histologic grade, or clinical size by accordance of Cox's proportional hazards regression model [11]. CD10 expression is associated with estrogen receptor-negativity, high tumour grade and decreased patient survival [10]. This protease has been shown to cleave doxorubicin, a key element in many cancer therapies, therefore its expression may correspond with chemoresistance. In this context, CD10 may be a potential target for anticancer therapy, as through inhibition of CD10's enzymatic activity, anti-tumour efficacy of well established chemotherapies may be enhanced [10]. In our study, the stromal : epithelial ratio for CD10 staining in non-cancerous FA was 40:100 compared with 15:95 in precancerous FA. In contrast to the literature's report of increased isolated stromal CD10 expression in cancerous lesions, CD10 positivity in epithelial-stromal crosstalk ratios show a decreasing trend in the precancerous test group of fibroadenomas. 
Bcl-2 (B cell lymphoma 2) is a member of the $b c l$ family of proteins responsible for regulating apoptosis. The $B C L 2$ gene acts as an oncogene in many solid organ tumours including breast cancer [12]. In normal breast tissue, $\mathrm{Bcl}-2$ is physiologically expressed in the ductal epithelial cells [13]. Bcl-2 is an apoptotic inhibitor and contributes to the control of both the cell cycle and cellular proliferation. Overexpression of Bcl2 causes cells to arrest in the G1 phase, halting the G0/G1-S phase transition [14].

$46 \%$ of breast ductal carcinomas are negative for Bcl-2 expression, whereas normal breast tissue is intensely positive [13]. In breast cancer patients, Bcl-2 overexpression is associated with improved prognostic features including a low mitotic count, estrogen-receptor positivity, high histological grade of differentiation, a low cell proliferation rate, tumour necrosis, and DNA diploidy [15]. In this context, highly proliferative breast tumours associated with a poor prognosis often have Bcl-2 downregulation [16]. Lobular breast cancers express moderate to strong Bcl-2 positivity more often (80\%) than ductal-type carcinomas (37\%). This may partially be explained by the discovery that there is a relative absence of $\mathrm{Bcl}-2$ proteins in poorly-differentiated cancers when compared to their well-differentiated counterpart, suggesting the protein is down-regulated as the tumour progresses [15]. The prognostic effect of Bcl-2, however, is only significant for the first five years after initial diagnosis, indicating its uses as a diagnostic tool are limited to this period of time. In a study of 5892 cases, Bcl2 was shown to be a significant prognostic marker independent of node status, tumour size and grade, and other biological variables. In addition, expression of Bcl-2 in these patients was associated with an increased overall and disease free survival [12]. In agreement with the literature, in our study expression of Bcl-2 was lower in the stroma of pre-cancerous lesions (20:80) when compared with the non-cancerous FAs (40:85). Decreased expression of this protein may be responsible for decreased apoptotic and cell-cycle control, resulting in an increased cellular proliferation.

CD31 (platelet endothelial cell adhesion molecule, PECAM-1), is a widely recognized angiogenic marker. In patients with DCIS, it is suggested that CD31 expression is indicative of cells attempting to enhance diffusion and progression [17]. DCIS with CD31 expression demonstrates a tendency to be of a higher nuclear grade, hormone independence, and lobular invasion [18]. Further, this adhesion molecule plays a role in organizing the architecture of developing breast carcinomas and in promoting cell differentiation through the establishment of cellular polarity. Finally, CD31 expression appears to be necessary for the development of motility, and therefore for tumour metastases [18]. CD31 has yet to be shown to be an independent prognostic factor and it therefore remains questionable whether CD31 is a cause or an effect of tumourigenesis [19]. Future investigations will help in directing the use of this receptor molecule in prevention and therapeutic management strategies. In our study, CD31 was not expressed in the epithelial component of either set of tissues; however, in the stroma expression dropped from an average of $30 \%$ to $10 \%$ in non-cancerous FA to pre-cancerous suggesting perhaps an altered signal direction indicative of stromal-epithelial dysfunction.

Nestin (neuroepithelial stem cell protein) is a type IV intermediate filament protein expressed in the beginnings of embryonic and fetal development by progenitor cells $[20,21]$. Upon differentiation, this protein is down-regulated and replaced with tissue-specific proteins. In the development of pathological conditions such as injury and neoplasia, it may be re-expressed [20]. Normally, nestin acts as a scaffold to maintain cell shape and tissue stability and was originally reported only in neuroepithelial stem cells; however, expression has since been described in gastrointestinal tract, pancreas, salivary gland, kidney, uterine cervix, endometrium, prostate, and breast [22]. Within the breast, nestin is expressed in the basal and myoepithelial layer of the mammary gland where it is can be used as a marker for the formation of endothelial or myoepithelial cells $[20,23,24]$. In addition, this protein has been found to be active in newly formed blood vessels [21].

Overexpression of nestin is most common in basal-like breast cancers, often in the triple-negative tumours (negative for estrogenreceptors, progestin-receptors or HER2) [20,24]. Nestin expression in breast cancer is correlated with increased rate of nodal metastasis and poor prognosis [24]. As such, overexpression of nestin has been suggested to be restricted to a very poorly-differentiated and highly aggressive tumour-type [23]. Due to a relative paucity of information about this protein, the specific role nestin plays in these malignant processes remains uncertain. As nestin is such a new protein to be studied, continued research is required to determine its specific functions and its utility in targeted detection and/or therapy. In our study, nestin positivity increased significantly in the stroma of precancerous FAs when compared with non-cancerous, with an S: E ratio of 40:80 and 15:80 respectively. Such findings agree with the literature that nestin has higher expression in neoplastic tissues, perhaps acting as an angiogenic factor.

In normal tissue, the gene TP53 encodes the p53 protein that is responsible for reproduction, genomic repair, metabolic processes, germ-cell fidelity and the surveillance of stem-cell development [25]. In the context of carcinogenesis, $\mathrm{p} 53$ responds to stress by binding to DNA to arrest of the cell-cycle, cause apoptosis/autophagy, and senescence [9]. The carcinogenic pathway may inactivate p53 by deletion of the TP53 gene, a missense mutation or inactivation of ARF gene [25]. Hundreds of genes are directly regulated by this protein [9] and therefore TP53 mutations may affect the expression of numerous protein-products. Though the literature reports mutations of stromal TP53 to be associated with increased loss of heterozygosity or allelic imbalances [26], in our study there was no significant change in stromal:epithelial ratios of $\mathrm{p} 53$ expression in the precancerous vs. noncancerous FA. We believe that p53 expression in the stroma is a delayed event in the multifactorial pathway of breast carcinogenesis. The strong expression of $\mathrm{p} 53$ in the atypical tumour-stromal fibroblasts that are recognized in invasive ductal carcinoma of the breast is not seen in our study as there is no recognizable alteration of the stromal fibroblasts which we hypothesize is a delayed event [3].

It has been suggested that the genetic alterations that occur in epithelial cells alone are insufficient in explaining the complex carcinogenesis that allows for the multiple phenotypic expressions of tumour cells and the complexity of tumour progression. The 'cross-talk' between these two elements is an ill-understood yet key line of communication that begins in normal breast during embryological development when the epithelial and mesenchymal cells communicate to ensure proper development and function [1]. The proliferation, survival, polarity, differentiation, and invasive capacity of mammary epithelia are modified by various stromal cells within the microenvironment [27]. Interestingly, these processes are important not only in the development of the normal breast but can reoccur after maturation, at which point they are considered mechanisms for tumourigenesis. Cross-talk between these two components may be by 
biochemical (signaling molecules) or physical (direct contact /via the extracellular matrix) means [28].

Though it has not yet been established whether epithelium or the stroma triggers cancer progression, the importance of both elements is increasingly being recognized. The microenvironment is believed to play a key role in the development of breast neoplasms as pictorially demonstrated in Figure 1. In normal tissue, the microenvironment is involved in reverting neoplastic changes through the initiation of cell differentiation [29]. Specific, regulated interaction between stromal and epithelial cells is necessary in the maintenance of non-neoplastic homeostasis [30]. The hostile environment characterized by oxidative stress, tissue hypoxia, nutrient deprivation, or changes in pressure or $\mathrm{pH}$ may leads to stromal instability [7]. Such volatility may result in the abnormal secretion of growth hormones, motility factors and extracellular molecules, altering this intricate communication [28]. Hypoxic conditions are associated with induction of genes involved in regulating cellular proliferation, the production of the ECM and cell adhesion. These conditions are responsible for limiting tumor cell division, selecting for malignant cells, and inducing adaptations promoting invasiveness [31].

Malfunctions in the interactivity between the stroma and the epithelial cells increase the probability of malignant transformation [32]. As such, cancer cells may evade the anti-tumourigenic signals from the microenvironment and continue to progress via the suppression of normal cells and the recruitment of cells with irregular functions. Eventually this can result in the destruction of the normal tissue architecture which can lead to neoplastic changes [6]. Once the epithelial involvement has been initiated these cells pass through a wellresearched progression starting with hyperplasia that develops into in situ and invasive cancer that may ultimately result in metastatic disease [33]. Such epithelial-stromal interactions were examined in a study investigating the role caveolin-1 (Cav-1) has on carcinogenesis. Cav1 is normally highly expressed in adipocytes and within the stroma. In this study, Williams et al found that loss of Cav-1 exacerbated mammary lobulo-alveolar hyperplasia and promoted growth of mammary tumour cells [34].

Various stromal elements may contribute to enhancing breast tumour progression and aggressiveness. Mathematical modeling and experimental data both suggest that conditions in the microenvironment such as hypoxia may provide selective pressure for the evolution of tumourigenesis [35]. Fibroblast, immune cell, growth factors and chemokines may all lead to stimulation of tumour cell growth and the recruitment of tumour precursors that then undergo abnormal proliferation [7]. Growth, differentiation, invasiveness, and polarity of epithelial cells have all been shown to be mediated by such stromal elements [29]. These factors thus create an environment permissive for tumourigenesis, as such cells possess characteristics and functions that have been altered to survive in the new conditions imposed by the tumour [32]. It is hypothesized that the proliferation, invasiveness, tumorigenicity, and potential for metastasis are all promoted through paracrine signalling between stromal and epithelial cells [35].

Fibroblasts are the major constituent of the stroma, responsible for the production of type IV collagen and laminin of the basement membrane and epithelial support $[7,36]$. In normal tissues, fibroblasts are involved in wound repair, in which they are activated and promote the secretion of ECM proteins and growth/chemotactic factors to coordinate both inflammatory and vascular progenitor cells. At the completion of healing, levels of these activated fibroblasts revert back to normal. By contrast in neoplastic tissue, cancer-associated fibroblasts (CAFs) are continually activated and resistant to apoptosis. Theories of CAF origins include epithelial cell transformation, local fibroblast activation, and bone-marrow cell alterations [7]. These cells show marked differences from their normal counterparts including greater expression of $\alpha$-smooth-muscle-actin ( $\alpha \mathrm{SMA}$ ), increased contractility, and a greater aptitude for promoting tumourgenesis by acting as a buffer to promote tumour growth [37]. Contractility is achieved by increased number of myofibroblasts in neoplastic tissue which are normally not present $[36,37]$.

The existence of an intimate cross-talk between development of the epithelium and blood vessels has been suggested, yet the relationship between the vascular supply and development of the mammary gland and adipogenic stroma remains uncertain [38]. The endothelial cells of the stroma are associated with angiogenesis, a process vital in tumour growth and survival [31]. In normal breast tissue, the endothelial cells contact the basement membrane and a layer of pericytes for support; during angiogenesis the basement membrane degrades while connections to the pericytes weaken, allowing endothelial cells to invade the surrounding stroma where they re-synthesize a basement membrane conducive to the acquisition of a capillary-like morphology. Vascular endothelial growth factor (VEGF) is a strong and selective endothelial mitogen, and the primary factor implicated in tumour vessel formations [39]. This chemical signal is directly secreted by both tumour and stromal cells (specifically fibroblasts, and inflammatory cells) [7]. VEGF acts on endothelial cell receptors and alters gene expression to promote cellular proliferation and increase capillary permeability [39]. Vessels formed by VEGF are atypical, with a non-uniform distribution within the tumour, an uncharacteristic shape, an unsuitable branching network, and have multiple blind ends [7]. The growth of these irregular vessels in a confined environment induces mechanical stress that compresses the lymph vessels, thereby facilitating lymphatic invasion and metastatic disease [40]. Tumour vessels that are incorrectly formed contribute to the creation of an unfavourable microenvironment, including hypoxia, acidosis, and increased interstitial fluid pressures, to which the tumour responds by expressing genes that alter cell growth, invasion, and metastases [7]. These three features are determinant of tumour growth, metabolism, and response to radiation and chemotherapy [40].

Despite advances in early detection by enhanced radiological imaging, the options available for patients presenting with advanced breast cancer remain limited [29]. The majority of therapeutic agents that target cancer cells have developed genetic instability and are prone to developing resistance. In this context, novel breast cancer markers and innovative therapeutic targets are required that include the tumour microenvironment and breast cancer stem cells as drug targets [41]. Stromal cells appear to be more stable and therefore the direction of future research [29]. A more thorough understanding of the components and interactions within the microenvironment may lead to the development of more accurate diagnostic tests and more efficient stromal therapeutic strategies. 
Citation: Kanthan R, Senger JL, Kanthan SC (2011) Stromal Signatures of Breast Carcinogenesis in Fibroadenoma—A Pilot Study. J Carcinogene Mutagene 2:123. doi:10.4172/2157-2518.1000123

Page 7 of 8

\section{Conclusions}

This preliminary study suggests early alterations in the stroma that may precede development of the epithelial phenotype of breast cancer. Such early changes may be due to alterations at a molecular/genetic/ epigenetic level similar to the multifactorial pathway of carcinogenesis as seen in colorectal carcinoma. Improved understanding of host stromal microenvironmental changes may result in the recognition of 'stromal signatures' as potential risk assessment markers in FA for breast carcinogenesis. Exploration of both benign and malignant breast pathologies in larger studies is necessary for validation of this hypothesis of 'stromal signatures' as risk assessment markers for breast cancer in men and women.

\section{Competing Interests}

The authors declare no competing interests and no financial interests

\section{Author's Contributions}

RK is the corresponding and first author of this manuscript.

JLS is a summer student who contributed to the acquisition, analysis, and interpretation of data.

SCK is the senior author

All authors have read and approved the final manuscript.

\section{Acknowledgements and Funding}

This project was completed with funding from the Canadian Breast Cancer Association.

\section{References}

1. Shekhar MP, Pauley R, Heppner G (2003) Host microenvironment in breas cancer development: extracellular matrix-stromal cell contribution to neoplastic phenotype of epithelial cells in the breast. Breast Cancer Res 5: 130-135.

2. El-Wakeel H, Umpleby HC (2003) Systematic review of fibroadenoma as a risk factor for breast cancer. Breast 12: 302-307.

3. Hasebe T, Iwasaki M, Akashi-Tanaka S, Hojo T, Shibata T, et al. (2011) Atypical tumor-stromal fibroblasts in invasive ductal carcinoma of the breast. Am J Surg Pathol 35: 325-336.

4. Hasebe T, Iwasaki M, Akashi-Tanaka S, Hojo T, Shibata T, et al. (2011) Important histologic outcome predictors for patients with invasive ductal carcinoma of the breast. Am J Surg Pathol 35: 1484-1497.

5. Hasebe T, Konishi M, Iwasaki M, Endoh Y, Nakagohri T, et al. (2005) Histological characteristics of tumor cells and stromal cells in vessels and lymph nodes are important prognostic parameters of extrahepatic bile duct carcinoma: a prospective study. Hum Pathol 36: 655-664

6. Erickson AC, Barcellos-Hoff MH (2003) The not-so innocent bystander: the microenvironment as a therapeutic target in cancer. Expert Opin Ther Targets 7: 71-88.

7. Li H, Fan X, Houghton J (2007) Tumor microenvironment: the role of the tumor stroma in cancer. J Cell Biochem 101: 805-815.

8. Sato Y, Goto Y, Narita N, Hoon DS (2009) Cancer cells expressing toll-like receptors and the tumor microenvironment. Cancer Microenviro 2: 205-214.

9. Lee EY, Muller WJ (2010) Oncogenes and tumor suppressor genes. Cold Spring Harb Perspect Biol 2: a003236.

10. Makretsov NA, Hayes M, Carter BA, Dabiri S, Gilks CB, et al. (2007) Stroma CD10 expression in invasive breast carcinoma correlates with poor prognosis, estrogen receptor negativity, and high grade. Mod Pathol 20: 84-89.

11. Iwaya K, Ogawa H, Izumi M, Kuroda M, Mukai K (2002) Stromal expression of CD10 in invasive breast carcinoma: a new predictor of clinical outcome. Virchows Arch 440: 589-593.

12. Callagy GM, Webber MJ, Pharoah PD, Caldas C (2008) Meta-analysis confirms
BCL2 is an independent prognostic marker in breast cancer. BMC Cancer 8 : 153.

13. Alireza A, Raheleh S, Abbass R, Mojgan M, Mohamadreza M, et al. (2008) An immunohistochemistry study of tissue Bcl-2 expression and its serum levels in breast cancer patients. Ann N Y Acad Sci 1138: 114-120.

14. Tsutsui S, Yasuda K, Suzuki K, Takeuchi H, Nishizaki T, et al.(2006) Bcl-2 protein expression is associated with p27 and p53 protein expressions and MIB-1 counts in breast cancer. BMC Cancer 6: 187.

15. Joensuu H, Pylkkanen L, Toikkanen S (1994) Bcl-2 protein expression and long-term survival in breast cancer. Am J Pathol 145: 1191-1198.

16. Tan PH, Bay BH, Yip G, Selvarajan S, Tan P, et al.(2005) Immunohistochemical detection of Ki67 in breast cancer correlates with transcriptional regulation of genes related to apoptosis and cell death. Mod Pathol 18: 374-381.

17. Sapino A, Bongiovanni M, Cassoni P, Righi L, Arisio R, et al. (2001) Expression of CD31 by cells of extensive ductal in situ and invasive carcinomas of the breast. J Pathol 194: 254-261.

18. Righi L, Deaglio S, Pecchioni C, Gregorini A, Horenstein AL, et al. (2003) Role of CD31/platelet endothelial cell adhesion molecule-1 expression in in vitro and in vivo growth and differentiation of human breast cancer cells. Am J Pathol 162: 1163-1174.

19. Dales JP, Garcia S, Carpentier S, Andrac L, Ramuz O, et al. (2004) Long-term prognostic significance of neoangiogenesis in breast carcinomas: comparison of Tie-2/Tek, CD105, and CD31 immunocytochemical expression. Human Pathol 35: 176-183.

20. Parry S, Savage K, Marchio C, Reis-Filho JS (2008) Nestin is expressed in basal-like and triple negative breast cancers. J Clin Pathol 61: 1045-1050.

21. Kolar Z, Ehrmann J Jr, Turashvili G, Bouchal J, Mokry J (2007)A nove myoepithelial/progenitor cell marker in the breast? Virchows Arch 450: 607609.

22. Kishaba Y, Matsubara D, Niki T (2010) Heterogeneous expression of nestin in myofibroblasts of various human tissues. Pathol Int 60: 378-385.

23. Li H, Cherukuri P, Li N, Cowling V, Spinella M, Cole M, Godwin AK, Wells W, DiRenzo J (2007) Nestin is expressed in the basal/myoepithelial layer of the mammary gland and is a selective marker of basal epithelial breast tumors. Cancer Res 67: 501-510.

24. Liu C, Chen B, Zhu J, Zhang R, Yao F, Jin F, Xu H, Lu P (2010) Clinical implications for nestin protein expression in breast cancer. Cancer Sci 101 815-819.

25. Lane D, Levine A (2011) p53 research: the past thirty years and the next thirty years. Cold Spring Harbor Laboratory Press 2: a000893.

26. Patocs A, Zhang L, Xu T, Weber F, Caldes T, et al. (2007) Breast-cance stromal cells with TP53 mutations and nodal metastases. N Engl J Med 357 2543-2551.

27. Polyak K, Kalluri R (2010) The role of the microenvironment in mammary gland development and cancer. Cold Spring Harb Perspect Biol 2: a003244.

28. Kim JB, Stein R, O'Hare MJ (2005) Tumour-stromal Interactions in breast cancer: The role of stroma in tumourgenesis. Tumour Biol 26: 173-185.

29. Allinen M, Beroukhim, Cai L, Brennan C, Lahti-Domenici J, Huang $\mathrm{H}$, et al (2004) Molecular characterization of the tumour microenvironment in breast cancer. Cancer Cell 6: 17-32.

30. Bierie B, Moses HL (2005) Under pressure: stromal fibroblasts change their ways. Cell 123: 985-987.

31. Finger EC, Giaccia A (2010) Hypoxia, inflammation, and the tumor microenvironment in metastatic disease. Cancer Metastasis Rev 29: 285-293.

32. Barcellos-Hoff MH, Medina D (2005) New highlights on stroma-epithelia interactions in breast cancer. Breast Cancer Res 7: 33-36.

33. Polyak K: Breast cancer (2007) origins and evolution. J Clin Invest 117: 31553163.

34. Williams TM, Sotgia F, Lee H, Hassan G, Di Vizio D, Bonuccelli G, Capozza 
Citation: Kanthan R, Senger JL, Kanthan SC (2011) Stromal Signatures of Breast Carcinogenesis in Fibroadenoma-A Pilot Study. J Carcinogene Mutagene 2:123. doi:10.4172/2157-2518.1000123

Page 8 of 8

et al. (2006) Stromal and epithelial caveloin-1 both confer a protective effect against mammary hyperplasia and tumorigenesis. Am J Pathol 169: 1784 1801.

35. Polyak K, Haviv I, Campbell IG (2002) Co-evolution of tumor cells and their microenvironment. Trends Genes 25(1): 30-38

36. Weinberg RA (2008) Coevolution in the tumor microenvironment. Nat Genet 40(5): 494-495.

37. Ostman A, Augsten M (2009) Cancer-associated fibroblasts and tumor growth - bystanders turning into key players. Current Opinion in Genetics \& Development 19: 67-73.
38. Wiseman BS, Web Z (2002) Stromal effects on mammary gland development and breast. Science 296: 1046-1049.

39. Boudreau N, Myers C (2003) Breast cancer-induced angiogenesis: multiple mechanisms and the role of the microenvironment. Breast Cancer Res 5: 140146.

40. Fukumura D, Jain RK (2007) Tumor microenvironment abnormalities: causes, consequences, and strategies to normalize. J Cell Biochem 101: 937-949.

41. Weigelt B, Bissell MJ (2008) Unraveling the microenvironmental influences on the normal mammary gland and breast cancer. Seminars in Cancer Biology 18: 311-321. 